\title{
Intra-arterial thrombolysis in acute ischemic stroke:A single center experience
}

\author{
Vikram Huded, Sachin Dhomne, Manish Shrivastava, Rashmi Saraf, Uday Limaye \\ Department of Radiology, Division of Interventional Neuroradiology, KEM Hospital, Mumbai, India
}

\author{
Address for correspondence: \\ Dr. Uday Limaye, \\ Division of Interventional \\ Neuroradiology, Department of \\ Radiology, KEM Hospital, \\ Mumbai - 400 012, India. \\ E-mail: udaylimaye123@gmail.com
}

DOI: $10.4103 / 0028-3886.59473$

\begin{abstract}
Intra-arterial thrombolysis (IAT) is a treatment modality in patients with acute large vessel occlusive ischemic stroke. To our knowledge, this is probably the first reported study of intra-arterial thrombolysis in acute ischemic stroke from India. Of the 17 patients treated who recieved IAT, successful recanalization was achieved in nine patients, Thrombolysis in Myocardial Infarction (TIMI) score of 2 or 3. At 90-day follow-up, eight patients achieved modified Rankin Scale (mRS) score of $<2$.
\end{abstract}

Key words: Acute stroke, intra-arterial thrombolysis, rtPA

\section{Introduction}

Intravenous thrombolysis (IVT) in acute ischemic stroke within three hours of onset has been an established treatment after the NINDS trial. ${ }^{[1]}$ The recent European Cooperative Acute Stroke Study (ECASS III) trial has extended the window period to four and half hours. ${ }^{[2]}$ In large vessel occlusions, early recanalization rates with IV rtPA are low, approximately $10 \%$ in internal carotid arteriy (ICA) occlusion and $30 \%$ in proximal middle cerebral artery (MCA) occlusion. ${ }^{[3,4]}$ Pro-Urokinase (r-proUK) for Acute Cerebral Thromboembolism (PROACT II) study suggests that intra-arterial thembolysis (IAT) can be given up to six hour window period in patients with middle cerebral artery occlusion and is an effective treatment but with some safety concerns. ${ }^{[5]}$ There is no published study from India on IAT in acute ischemic stroke. Even though about 2500 patients with stroke are admitted yearly in our institute only a handful of patients present within the window period. The overall purpose of this paper is to report the summary of IAT in acute stroke in a tertiary referral center from India.

\section{Materials and Methods}

This is a retrospective study of 17 patients with acute ischemic stroke treated by intra-arterial thrombolysis (IAT) between 1999 and 2009 with at least 3-month follow-up. The clinical inclusion criteria were age between 18 and 85 years, focal neurological deficits consistent with stroke within six hours of onset, measurable neurological deficit - National Institute of Health Stroke Scale (NIHSS) score more than or equal to four, and informed consent by either the patient or care giver. Clinical exclusion criteria included rapidly improving neurological signs, an NIHSS score greater than 30, history of stroke within the previous six weeks, seizures at onset, previous history of intracranial hemorrhage at any time, neoplasm, or subarachnoid hemorrhage, surgery within 30 days, head trauma within 90 days, active or recent hemorrhage within 30 days, known hemorrhagic diathesis, baseline international normalized ratio greater than 1.7 , activated partial thromboplastin time more than 1.5 times the normal, or baseline platelet count less than $1,00,000 /$ c.c. Computed tomographic (CT) scan exclusion criteria were intracranial tumors, hemorrhage, significant mass effect with midline shift, and acute hypodense parenchymal lesion or effacement of cerebral sulci in more than one-third of the vascular territory. Patients who met all clinical and CT scan criteria were taken for four-vessel diagnostic cerebral angiography after taking consent from patient/relatives. The patients were taken up for IAT if there was a large vessel occlusion with Thrombolysis in Myocardial Infarction (TIMI) score of zero or one. Patients received $3000 \mathrm{U}$ of heparin bolus. A 6 F guiding catheter was placed into the symptomatic artery. Microcatheter was then navigated into the thrombus. Intra-arterial infusion of Urokinase (UK) or rtPA was performed in small doses and repeated until the maximum dose was reached (rtPA $40 \mathrm{mg}$ and Urokinase 1.2 million I.U.), complete recanalization (TIMI 2 or 3 ) was achieved or time after onset was more than eight 
hours. Since the last five years we have been using rtPA as it is highly fibrin specific and recanalization is better with rtPA compared to UK. ${ }^{[6]}$

CT scan was done post thrombolysis and at 24 hours. It was repeated if patient's condition deteriorated at any time during follow-up. NIHSS score and modified Rankin Scale (mRS) score were documented at 24 hours, 30 days and 90 days. All causes of mortality and procedure-related complications were analyzed. The primary outcome was percentage of patients achieving mRS of two or less at 90 days follow-up. The secondary outcome was percentage of patients achieving complete angiographic recanalization and also the percentage of patients who presented within 3 hours duration achieving mRS 0-1 at 90 days.

\section{Results}

During the study period 17 patients with acute stroke had IAT [Table 1]. The mean age was 55.3 years (range 37 to 85 years) and eleven were males. The mean duration of presentation was 3.35 hours (range 0.5 hours to 6 hours). The median NIHSS score was 17 (range 10 to 22). Twelve patients had middle cerebral artery (MCA), three had carotid ' $\mathrm{T}$ ' and two had basilar artery (BA) occlusions. Nine (53\%) patients had TIMI 2 or 3 recanalization rate and eight $(47.06 \%)$ patients had mRS score of $<2$. There were three deaths and the causes of death include intracerebral hemorrhage (1), massive edema and herniation (1), and reocclusion of the artery and brainstem infarct (1). We had eight patients who presented within three hours duration. The mean age of these patients was 59.12 years (range 42 to 75 years) and four were male.
The mean duration of presentation was 1.75 hours (range 0.5 hours to 3 hours) and the median NIHSS score was 16 (range 10 to 22). Five patients had MCA, two patients had Carotid ' $T$ ' and one had BA occlusions. There was no mortality and none of the patients had intracranial hemorrhage. Seven $(87.5 \%)$ patients achieved $\mathrm{mRS}$ score of 0 to 2 at 90 days, four (50\%) patients achieved mRS of 0 to 1 at 90 days and six (75\%) patients had TIMI 2 or 3 recanalization rate [Figures 1 and 2].

We had 12 patients who presented with MCA occlusion [Figures 3-5]. The mean age of patients was 53.41 years (range 43 to 75 years) and ten were males. The mean duration of presentation was 3.5 hours (range 0.5 hours to 6 hours) and the median NIHSS score was 17 (range 10 to 22). One patient had intracranial hemorrhage. Five $(41.66 \%)$ patients achieved mRS score of 0 to 2 at 90 days and six $(50 \%)$ patients had TIMI 2 or 3 recanalization rate.

\section{Discussion}

The efficacy of IVT in acute ischemic stroke has been proven in NINDS trial. ${ }^{[1]}$ When the NINDS trial was further analyzed with respect to stroke severity, the degree of benefit declined with increasing NIHSS scores. Patients with NIHSS scores of more than 20 had $6 \%$ absolute risk reduction in achieving $\mathrm{mRS}$ score of $<1$ at 90 days. If all the presently available data were analyzed, the efficacy of recanalization after IVT of patients with large arteries occlusion (internal carotid artery, MCA - M1 segment, or BA especially proximal BA) would be $30 \%$ compared to 60 to $70 \%$ in IAT. ${ }^{[7]}$ The efficacy of IVT in large vessel occlusions is lesser compared to IAT. In a large retrospective study, comparing IA and IV treatments

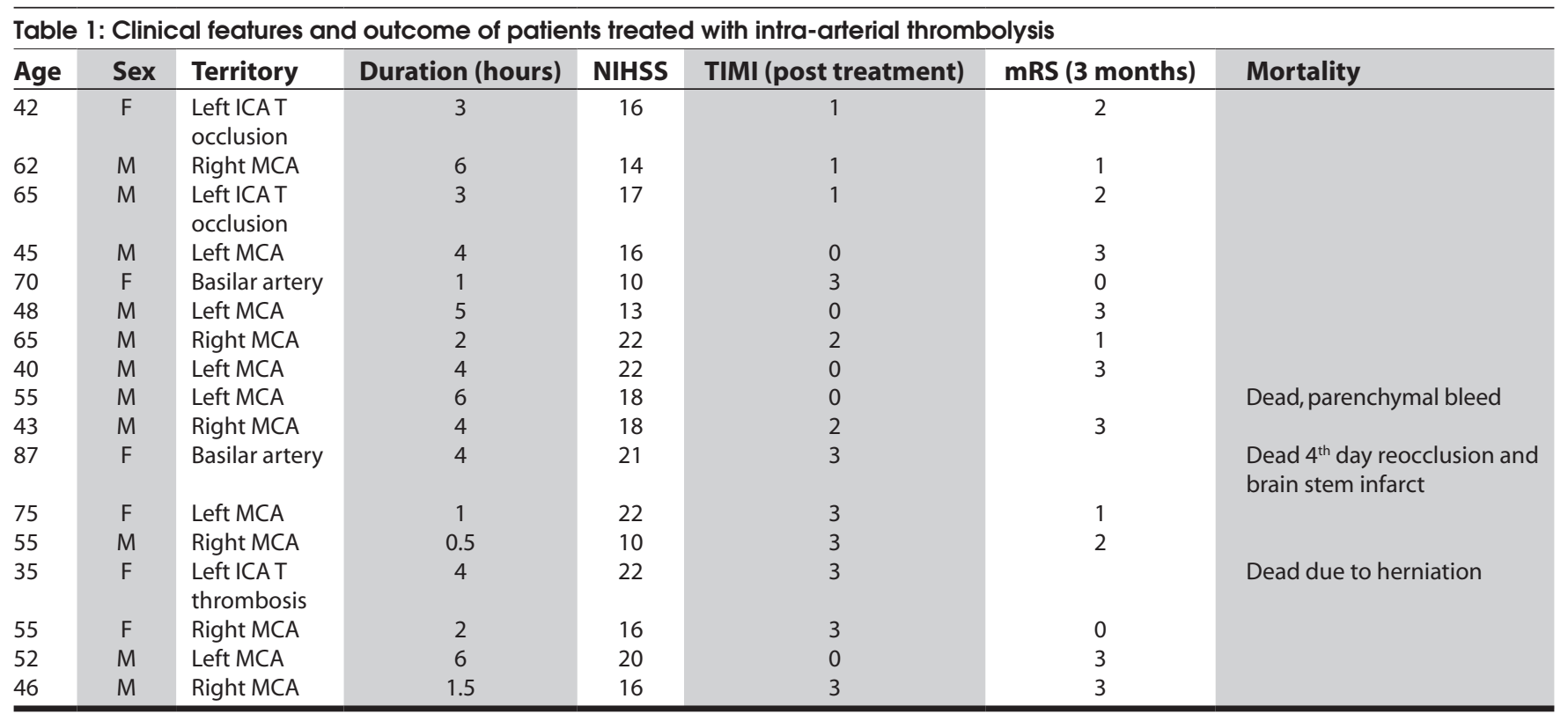




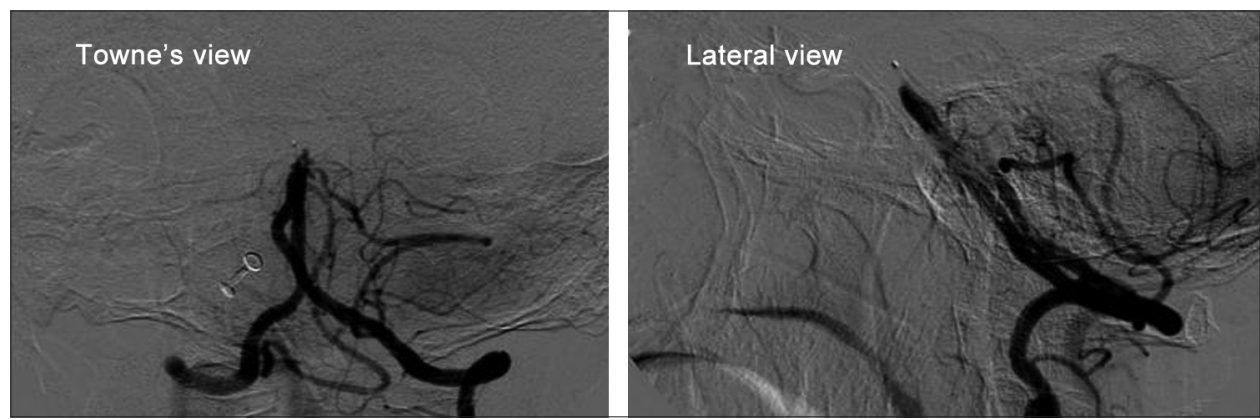

Figure 1: Vertebral angiogram showing basilar artery thrombosis

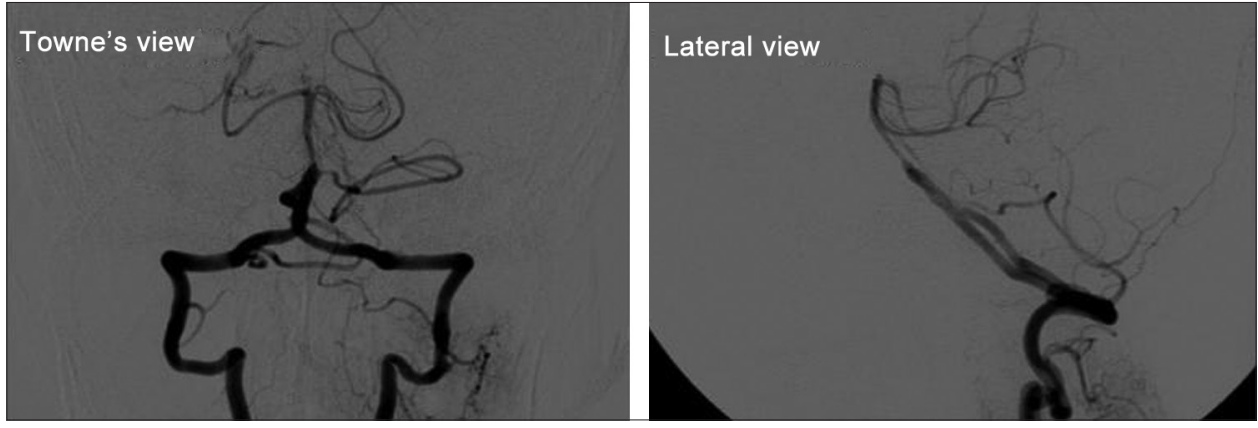

Figure 2: Post thrombolysis angiogram showing complete recanalization of basilar artery (TIMI 3)

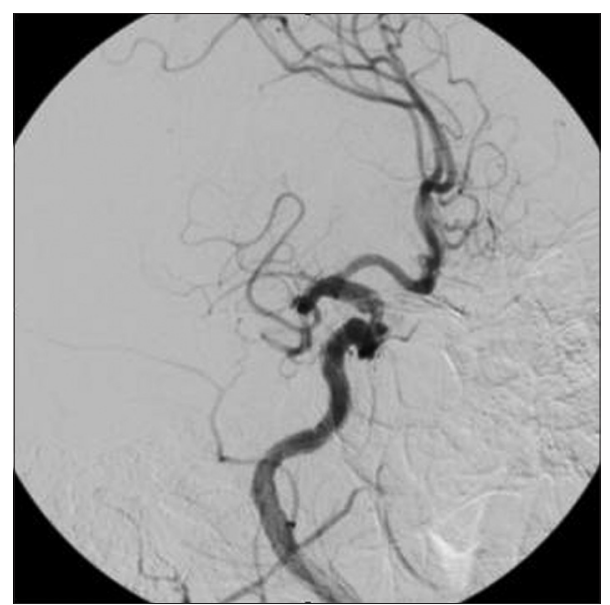

Figure 3: Right internal carotid angiogram showing middle cerebral artery occlusion

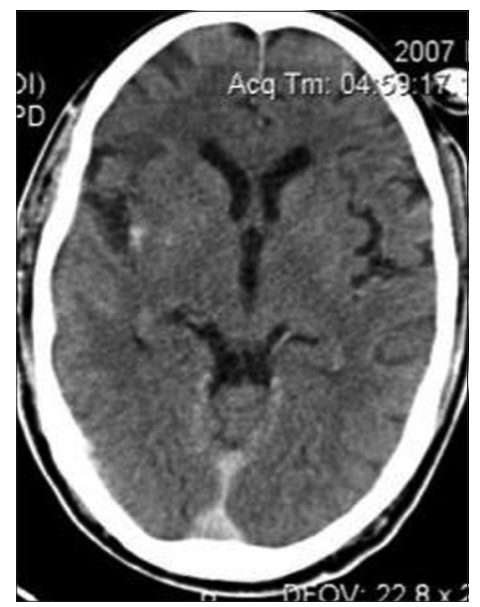

Figure 5: Post thrombolysis computed tomographic scan shows no hemorrhage

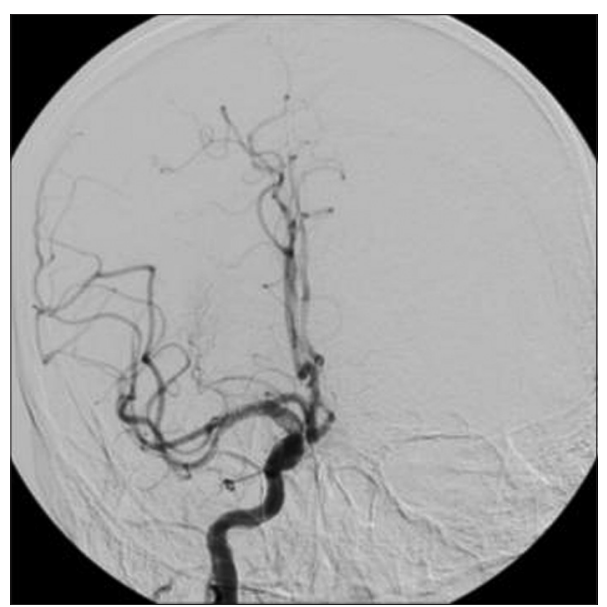

Figure 4: After $40 \mathrm{mg}$ of rtPA, complete recanalization of middle cerebral artery (TIMI 3 ) was achieved

in $\mathrm{M} 1$ occlusion presenting within 3 hours matched for age and stroke severity, favorable outcome was more frequent after IAT than after IVT ( $53 \%$ vs. $23 \%$; $P=0.001)$, and mortality was lower after IAT than after IVT (7\% vs. $23 \% ; P=0.022)$. IA treatment was associated with a significantly better outcome despite the longer 'door to needle time' ${ }^{\text {. }}{ }^{[8]}$ Efficacy of combined IV and IA treatment will be assessed by the ongoing Interventional Management Study III.

There are two randomized trials of IAT which included patients with MCA occlusion. In PROACT II trial, 40\% of r-prourokinase group and $25 \%$ of control patients had $\mathrm{mRS}$ score of $<2$. The recanalization rate was $66 \%$ for r-prourokinase group. The mortality was $25 \%$ for the r-proUrokinase group and $27 \%$ for the control group. ${ }^{[5]}$ In 
the Middle Cerebral Artery Embolism Local Fibrinolytic Intervention Trial (MELT) trial, $49.1 \%$ of urokinase group and $38.6 \%$ of control patients had a mRS score of $<2$. Even though they have not used TIMI score, 73.7\% of patient achieved partial or complete recanalization in urokinase group. The mortality was $5.3 \%$ in the urokinase group and 3.5\% in the control group at three months and intracerebral hemorrhage within 24 hours of treatment occurred in 9 and $2 \%$, respectively. ${ }^{[9]}$ In the analysis of studies of IAT in patients with acute ischemic stroke, there were more favorable outcomes in the IAT than the control group ( $41.5 \%$ vs. $23 \%, P=0.002)$, with a lower mortality rate for IAT (27.2\% vs. $40 \%, P=0.004)$. Symptomatic intracerebral hemorrhage was more frequent in the IAT group compared with the control group $(9.5 \%$ vs. $3 \%, P=0.046) .{ }^{[10]}$

Despite the advantages, there are limitations to the IAT. These include availability of specialized equipment, experienced trained personnel and costs. In our study, the recanalization rate was $53 \%$ (TIMI 2 or 3 ). $47 \%$ patient had mRS score of $<2$ at three months. When we analyzed the patients with MCA occlusion; $42 \%$ of patients had a favorable outcome and the recanalization was $50 \%$. Symptomatic cerebral hemorrhage occurred in one patient. In BA occlusion; one of the two patients had a favorable outcome. In ICA T occlusion; one of the three patients had a favorable outcome. In the study by Gonner et al. the recanalization rate was $63 \%$ and $21 \%$ of patients recovered to mRS score of 0 or 1 and $40 \%$ patients to mRS score of 2 or 3 . Outcome was good (mRS 0-3) in $80 \%$ of patients with MCA occlusions, $33 \%$ with ICA, and in 50\% with BA occlusion. Symptomatic cerebral hemorrhage occurred in $4.7 \%$ of patients. ${ }^{[1]}$

Our study has many limitations like small cohort, retrospective collection of data, non randomized design, single center involvement and non-blinded nature of the study. Our study suggests that intra-arterial thrombolysis is a feasible option in the subgroup of patients with large vessel occlusion. There, however, is a need for a randomized multicentric study to support our results.

\section{References}

1. Tissue plasminogen activator for acute ischemic stroke. The National Institute of Neurological Disorders and Stroke rt-PA Stroke Study Group. N Engl J Med 1995;333:1581-7.

2. Hacke W, Kaste M, Bluhmki E, Brozman M, Dávalos A, Guidetti D, et al. Thrombolysis with Alteplase 3 to 4.5 Hours after acute ischemic stroke. N Engl J Med 2008;359:1317-29.

3. del Zoppo GJ, Poeck K, Pessin MS, Wolpert SM, Furlan AJ, Ferbert A, et al. Recombinant tissue plasminogen activator in acute thrombotic and embolic stroke. Ann Neurol 1992;32:78-86.

4. Wolpert SM, Bruckmann H, Greenlee R, Wechsler L, Pessin MS, del Zoppo G.J. Neuroradiologic evaluation of patients with acute stroke treated with recombinant tissue plasminogen activator. The rt-PA Acute Stroke Study Group. AJNR Am J Neuroradiol 1993;14:3-13.

5. Furlan A, Higashida R, Wechsler L, Gent M, Rowley H, Kase C, et al Intra-arterial prourokinase for acute ischemic stroke.The PROACTII study: A randomized controlled trial. Prolyse in Acute Cerebral Thromboembolism. JAMA 1999;282:2003-11.

6. Eckert B, Kucinski T, Pfeiffer G, Groden C, Zeumer H. Endovascular therapy of acute vertebrobasilar occlusion: Early treatment onset as the most important factor. Cerebrovasc Dis 2002;14:42-50.

7. Moonis M. Intraarterial thrombolysis within the first three hours after acute ischemic stroke in selected patients. Stroke 2009;40:2611-2.

8. Mattle HP, Arnold M, Georgiadis D, Baumann C, Nedeltchev K, Benninger $\mathrm{D}$, et al. Comparison of intraarterial and intravenous thrombolysis for ischemic stroke with hyperdense middle cerebral artery sign. Stroke 2008;39:379-83

9. Ogawa A, Mori E, Minematsu K, Taki W, Takahashi A, Nemoto S, et al. Randomized trial of intraarterial infusion of urokinase within 6 hours of middle cerebral artery stroke. The middle cerebral artery embolism local fibrinolytic intervention trial (MELT) Japan. Stroke 2007;38:2633-9.

10. Lisboa RC, Jovanovic BD, Alberts MJ. Analysis of the safety and efficacy of intra-arterial thrombolytic therapy in ischemic stroke. Stroke 2002;33:2866-71.

11. Gönner F, Remonda L, Mattle H, Sturzenegger M, Ozdoba C, Lövblad KO, et al. Local intra-arterial thrombolysis in acute ischemic stroke. Stroke 1998;29:1894-900.

Accepted on 02-11-2009

Source of Support: Nil, Conflict of Interest: None declared. 